\title{
A GC-MS based metabolic profiling of fermented tomato by lactic acid bacteria
}

\author{
Eun-Ju Kim ${ }^{1 \dagger}$, Seong-Eun Park ${ }^{1 \dagger}$, Seung-Ho Seo ${ }^{1}$, Oh-Cheol Kweon ${ }^{2}$ and Hong-Seok Son ${ }^{1 *}$ (1)
}

\begin{abstract}
A GC/MS-based metabolite profiling was performed to investigate metabolic differences of fermented tomatoes according to the inoculation of different LAB strains. PCA score plot derived from 2554 signal features of GC-MS data and PCA biplot derived from 18 identified metabolites showed clear separation into three groups. Citric acid and malic acid were found to affect groups clustered with Lactobacillus fermentum (LF), Bifidobacterium longum (BL), and Pediococcus pentosaceus (PP) whereas lactic acid, succinic acid, and fructose were related to Lactobacillus plantarum $(L P)$ and Leuconostoc mesenteroid (LM) groups. Meanwhile, Lactobacillus brevis (LB) was associated with erythritol. Aminoacyl-tRNA biosynthesis and metabolism of cysteine and methionine were identified as metabolic pathways affected by the use of different $L A B$ groups ( $L F, B L$, and $P P \vee s$. $\angle B$ groups). This study highlights the applicability of metabolic profiling for understanding fermentative characteristics of $L A B$ strains.
\end{abstract}

Keywords: Fermentation, GC-MS, Lactic acid bacteria, Metabolomics, Tomato

\section{Introduction}

Tomatoes are among the most consumed agricultural products worldwide. They are rich in antioxidant compounds such as carotenoids, lycopene, vitamin $\mathrm{C}$, and vitamin E [1-3]. Although some tomato products such as paste, juice, and sauce are processed before consumption, tomatoes are generally consumed fresh because they have a short shelf-life due to rapid microbial spoilage. Fermentation is a simple and valuable technique to extend shelf-life properties. Fermentation can also meet the demands of consumers for non-dairy beverages with high nutritional value, vegetarians, and lactose intolerant individuals [4]. Recently, studies on tomato fermentation as a method to improve its antioxidant activity have been reported $[5,6]$.

Microorganisms can degrade organic substances with their own enzymes and many metabolic changes occur during fermentation. New molecular approaches are needed to gain new insights and to control fermentation

\footnotetext{
*Correspondence: hsson@dsu.ac.kr

${ }^{\dagger}$ Eun-Ju Kim and Seong-Eun Park are co-first authors who contributed equally to this work

1 School of Korean Medicine, Dongshin University, Naju 58245, Republic of Korea

Full list of author information is available at the end of the article
}

processes. The field of metabolomics involves holistic analysis of metabolic changes in the complete set of small compound [7]. One advantage of the metabolic approach is fingerprinting, which is largely used to monitor metabolic patterns associated with changing metabolites [8]. Metabolomics studies have been applied to investigate the fermentation of vegetables and fruits, such as beet root, cucumbers, and pineapples [9-11]. However, little is known about metabolic changes during tomato fermentation.

The ability of fermentation depends on the type of microbial starter used in the fermentation. For example, allochthonous strains showed delayed growth phases during tomato fermentation compared to selected autochthonous strains from tomatoes [12]. Some studies have shown that total antioxidant and ACE inhibition activities were different, depending on the lactic acid bacteria (LAB) strains [13]. Lactobacillus plantarum, Lactobacillus brevis, and Pediococcus pentosaceus were the main $\mathrm{LAB}$ strains isolated from spontaneously-fermented tomatoes [4]. The use of different LAB strains also affects the final metabolites in fermented tomatoes. The relationship between LAB strains and metabolic differences in fermented tomatoes is currently unclear. Thus, the objective of this study was to determine the 
metabolic differences of fermented tomatoes inoculated with different LAB strains, using GC-MS data sets coupled with multivariate analysis.

\section{Materials and methods Tomato preparation}

Tomatoes used in this study were purchased from a local market (Naju, Jeollanam-do) in Korea. Total soluble solids ( $\left.{ }^{\circ} \mathrm{Brix}\right)$ of these tomatoes showed $5.2^{\circ} \mathrm{Brix}$. The $\mathrm{pH}$ of these tomatoes and total acidity as lactic acid were 4.26 and $1.40 \%$, respectively. Tomatoes were ground, mixed, and diluted with distilled water at a ratio of 1:1. Fermentation for each of the six LAB strains was performed to five replications at $37{ }^{\circ} \mathrm{C}$ for $72 \mathrm{~h}$. The amounts of tomato samples in each fermentation were $500 \mathrm{~mL}$.

\section{Culture condition}

Lactobacillus plantarum (KCCM 11322, LP), Lactobacillus fermentum (KCCM 40401, LF), and Lactobacillus brevis (KCCM 11904, LB) were obtained from KCCM (Seoul, Korea). Leuconostoc mesenteroid (KCTC 3718, LM), Pediococcus pentosaceus (KCTC 3116, PP), and Bifidobacterium longum (KCTC 3128, BL) were obtained from KCTC (Daejeon, Korea). One percent (v/v) of pre-cultured $\mathrm{LAB}$ starter was inoculated for tomato fermentation.

\section{Total phenol content, flavonoid content, and DPPH scavenging activity}

Total phenol contents were analyzed by a modified Folin-Denis method [14]. Briefly, $0.5 \mathrm{~mL}$ of each sample was added to distilled water $(4.5 \mathrm{~mL})$ and Folin-Ciocalteu's phenol reagent $(0.5 \mathrm{~mL})$. Five $\mathrm{mL}$ of $7 \% \mathrm{Na}_{2} \mathrm{CO}_{3}$ was then added and the mixture was left at room temperature for $90 \mathrm{~min}$. The absorbance at $750 \mathrm{~nm}$ was measured.

Total flavonoid contents were measured by the modified method of Zhishen et al. [15]. Briefly, each sample $(1 \mathrm{~mL})$ was added to distilled water $(4 \mathrm{~mL})$ and $\mathrm{NaNO}_{2}$ $(0.3 \mathrm{~mL})$. After $5 \mathrm{~min}, 10 \% \mathrm{AlCl}_{3}(0.3 \mathrm{~mL})$ was added to the mixture. Next, $2.4 \mathrm{~mL}$ of distilled water and $2 \mathrm{~mL}$ of $1 \mathrm{~N} \mathrm{NaOH}$ were added. The absorbance was measured at $510 \mathrm{~nm}$.

DPPH radical scavenging activities were measured by the Blois method [16], with slight modification. Briefly, $0.4 \mathrm{~mL}$ of each sample was added to $1.6 \mathrm{~mL}$ of $0.4 \mathrm{mM}$ 1,1-diphenyl-2-picryl-hydrazyl. The absorbance at $525 \mathrm{~nm}$ was then measured.

\section{GC-MS analytical method}

Analytical methods and conditions associated with GCMS analysis were similar to those described in previous studies $[17,18]$ with minor modifications. Briefly, tomato samples were centrifuged at 13,000 rpm for $5 \mathrm{~min}$, then
$100 \mu \mathrm{L}$ of sample supernatant was lyophilized. Next, freeze-dried tomato samples were methoxymated using methoxyamine in pyridine solution, and trimethylsilylated by BSTFA (containing 1\% TMCS). Next, methyl stearate in heptane was added as an internal standard. To monitor the analytical variability, quality control (QC) samples were prepared by pooling equal volumes (approximately $10 \mu \mathrm{L}$ ) of each sample prior to the derivatization process. QC samples were analyzed every 10 samples throughout the GC-MS analysis. One milliliter of heptane was added into an Eppendorf tube as a blank sample.

Derivatized samples were injected into a QP-2020 Gas Chromatography Mass Spectrometer (Shimadzu, Kyoto, Japan) through a Rtx-5MS capillary column $(30 \mathrm{~m} \times 0.25 \mathrm{~mm})$, with a film thickness of $0.25 \mu \mathrm{m}$. The Oven temperature was programed at $60^{\circ} \mathrm{C}$ for $1 \mathrm{~min}$, then increased to $280{ }^{\circ} \mathrm{C}$ by $10{ }^{\circ} \mathrm{C}$ per min, and maintained at $280{ }^{\circ} \mathrm{C}$ for $10 \mathrm{~min}$. Temperatures of the injector, the transfer line, the ion source, and the quadrupole were set at $250,280,230,150{ }^{\circ} \mathrm{C}$, respectively. The MS was programmed in full scan mode at 50 to $550 \mathrm{~m} / \mathrm{z}$, and the electron impact of ionization was set at $70 \mathrm{eV}$.

\section{Data processing and multivariate analysis}

GC-MS raw data were subjected to XCMS web software (https://xcmsonline.scripps.edu) for baseline correction, noise removal, and alignment. Parameters of the basic centWave method for the GC single quadruple were set as described previously [18]. They were: signal/noise threshold, 2; mzdiff, 0.1; integration methods, 1; prefilter peaks, 3; prefilter intensity, 10000; mzwid, 0.25; minfrac, 0.5 ; and bandwidth, 3 . Next, values were corrected by subtracting the average of the blank sample at each feature. Feature intensities were normalized according to the intensity of methyl stearate prior to multivariate statistical analyses. Principal component analysis (PCA) was performed using SIMCA-P 154.0 software (Umetrics, Umea, Sweden). Biplot of PCA and pathway analysis were generated using the web-based MetaboAnalyst 4.0 (http://www.metaboanalyst.ca). Metabolites were identified based on similarity of RT (retention time), RI (retention index of $n$-alkane), mass spectrum (NIST 14.0 library), and in-house library data, using similar analytical method [17]. The similarity value (\%) was calculated by the similarity of mass spectrum of a metabolite in NIST library and QC sample.

\section{Metabolic pathway analysis}

Metabolic pathways and pathway topological analyses were conducted using MetaboAnalyst 4.0. 


\section{Results}

\section{Physicochemical and antioxidant properties of fermented tomatoes}

Changes in physicochemical and antioxidant properties of tomatoes fermented with six-different LAB strains are presented in Fig. 1. After 3 days of fermentation, soluble solid contents decreased to $1.42-1.82^{\circ} \mathrm{Brix}$. The $\mathrm{pH}$ value and TA in tomatoes before inoculation were 4.26 and $0.7 \%$, respectively. Differences in soluble solid contents, $\mathrm{pH}$, and TA were dependent on the fermentation strains used, indicating that the quality of fermented tomatoes varied depending on the LAB strain used.

Total phenol and flavonoid contents were increased in tomatoes fermented by $L M$ and $L P$, respectively. These fermented tomatoes also showed higher DPPH radical scavenging activities compared to other samples, suggesting that some new antioxidant components might have been produced during the fermentation of tomatoes by $L M$ and $L P$. Differences in phenolic compounds after fermentation might be associated with changes in the
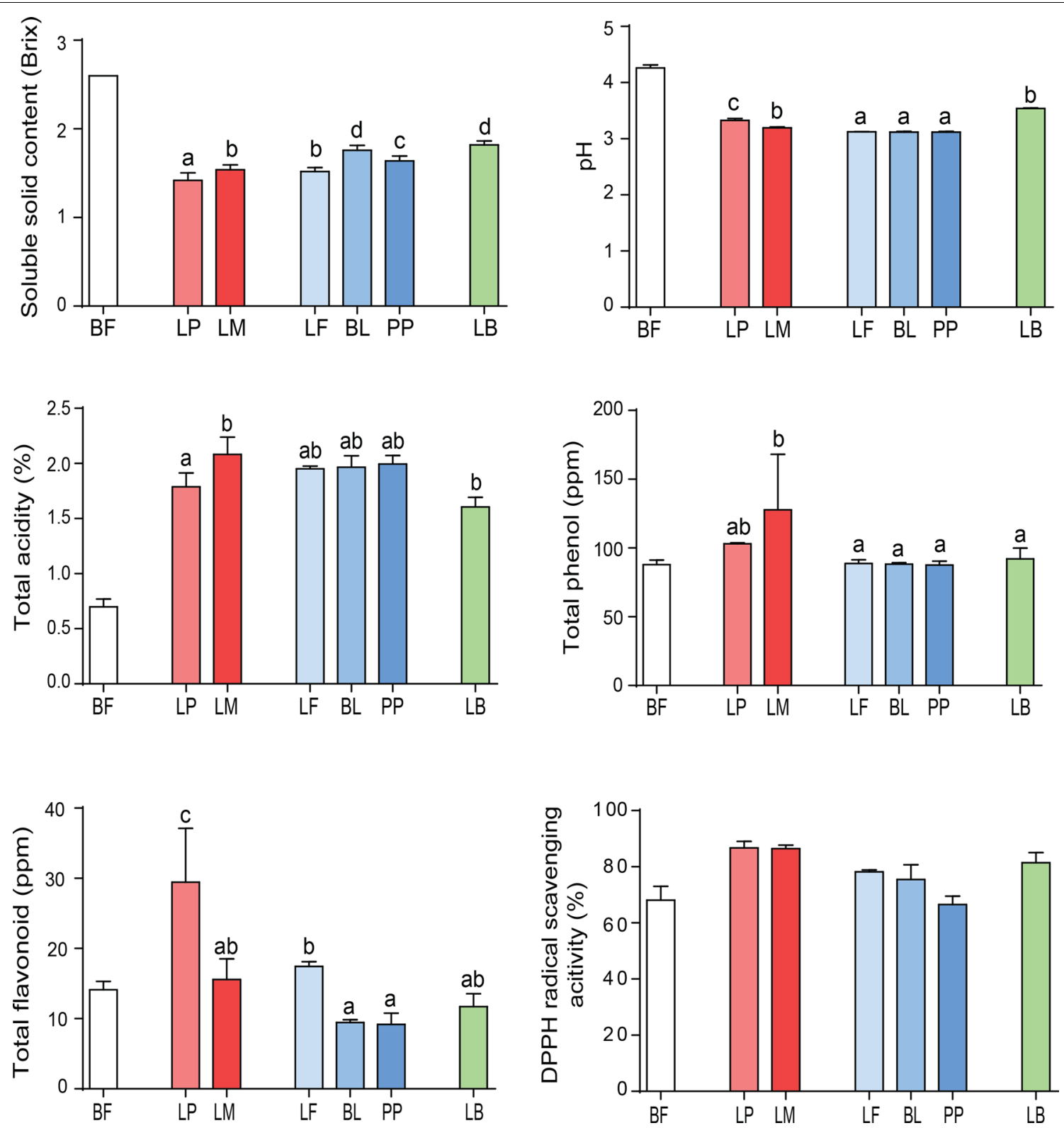

Fig. 1 Descriptive physicochemical and antioxidant properties of tomato samples fermented with different $L A B$ strains. Means followed by different letters are significantly different between samples $(p<0.05)$. BF before fermentation; $L P$ Lactobacillus plantarum; $L M L$ Leuconostoc mesenteroid; $L F$ Lactobacillus fermentum; BL Bifidobacterium longum; PP Pediococcus pentosaceus; LB Lactobacillus brevis 
sensory characteristics of foods such as color and flavor as well as differences in antioxidant effects [19].

\section{Metabolites changes of fermented tomatoes}

Tomato metabolites were analyzed using GC-MS to determine metabolic changes in the fermented tomatoes and the effect of different LAB strains. After data processing using XCMS, a total number of 2554 signal features were obtained. PCA score plot derived from the GC-MS data of the tomatoes is shown in Fig. 2a. There was a clear separation by PC1 between tomato samples before and after fermentation. Cumulative $R^{2} X$ and $Q^{2}$ values were 0.663 and 0.635 , respectively. These results indicated dramatic metabolic changes after 3 days of fermentation by LAB.

Among the 2554 features detected by GC-MS, a total of 18 metabolites were identified. Table 1 summarizes the metabolites identified in this study and their changes after fermentation. Levels of lactic acid, succinic acid, alanine, methionine, aspartic acid, glutamic acid, and erythritol were increased, while levels of malic acid, citric acid, serine, threonine, and fructose were decreased after tomato fermentation. However, changes in some amino acids, such as valine, leucine, and phenylalanine, showed different patterns with different LAB strains.

\section{Metabolite profiling of fermented tomatoes by different LABs}

To determine metabolic differences of tomatoes fermented by the different LAB strains used, PCA modeling was performed on samples after fermentation without samples before fermentation (Fig. 2b). Interestingly, PCA score plot showed a clear separation into three groups. The first group included tomatoes fermented with $P P$, $L F$, and $B L$. These samples were located close to each other in the PCA score plot, indicating that the metabolic profiles of these samples were more similar than those of other samples. The second group included tomatoes
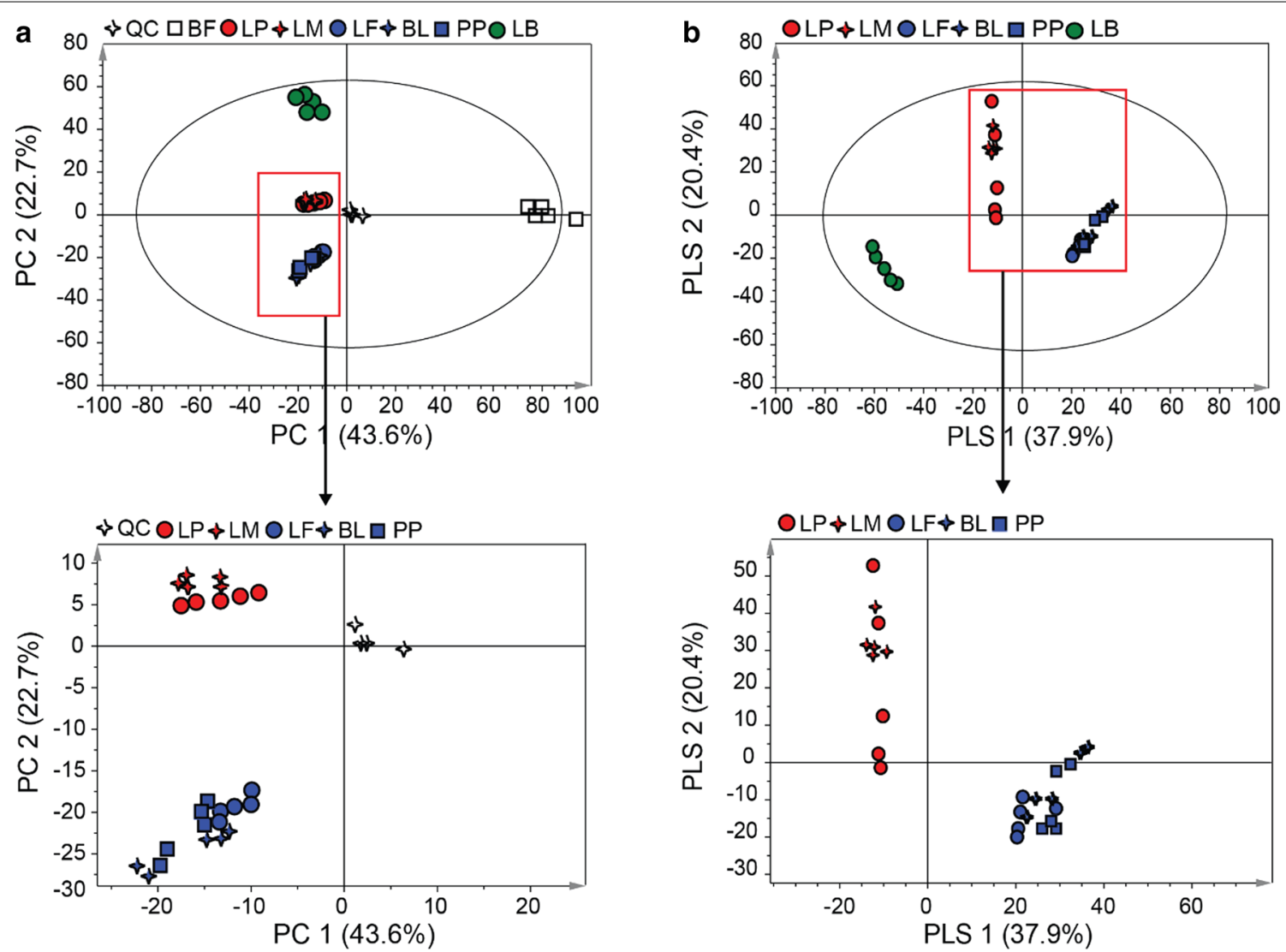

Fig. 2 PCA score plot (a) derived from GC-MS data of tomato samples fermented with different LAB strains, showing different metabolic profiles after 3 days of fermentation. QC (quality control) samples had good clustering in the center of PCA score plot, ensuring reliability of the metabolomics analysis. The score plot of panel (b) was generated after excluding samples before fermentation and QC samples in order to clarify metabolite similarity between $L A B$ strains. Each symbol in the score plot represents a tomato sample fermented in a different batch. $B F$ before fermentation, LP Lactobacillus plantarum, LM Leuconostoc mesenteroid, LF Lactobacillus fermentum, BL Bifidobacterium longum, PP Pediococcus pentosaceus, LB Lactobacillus brevis 


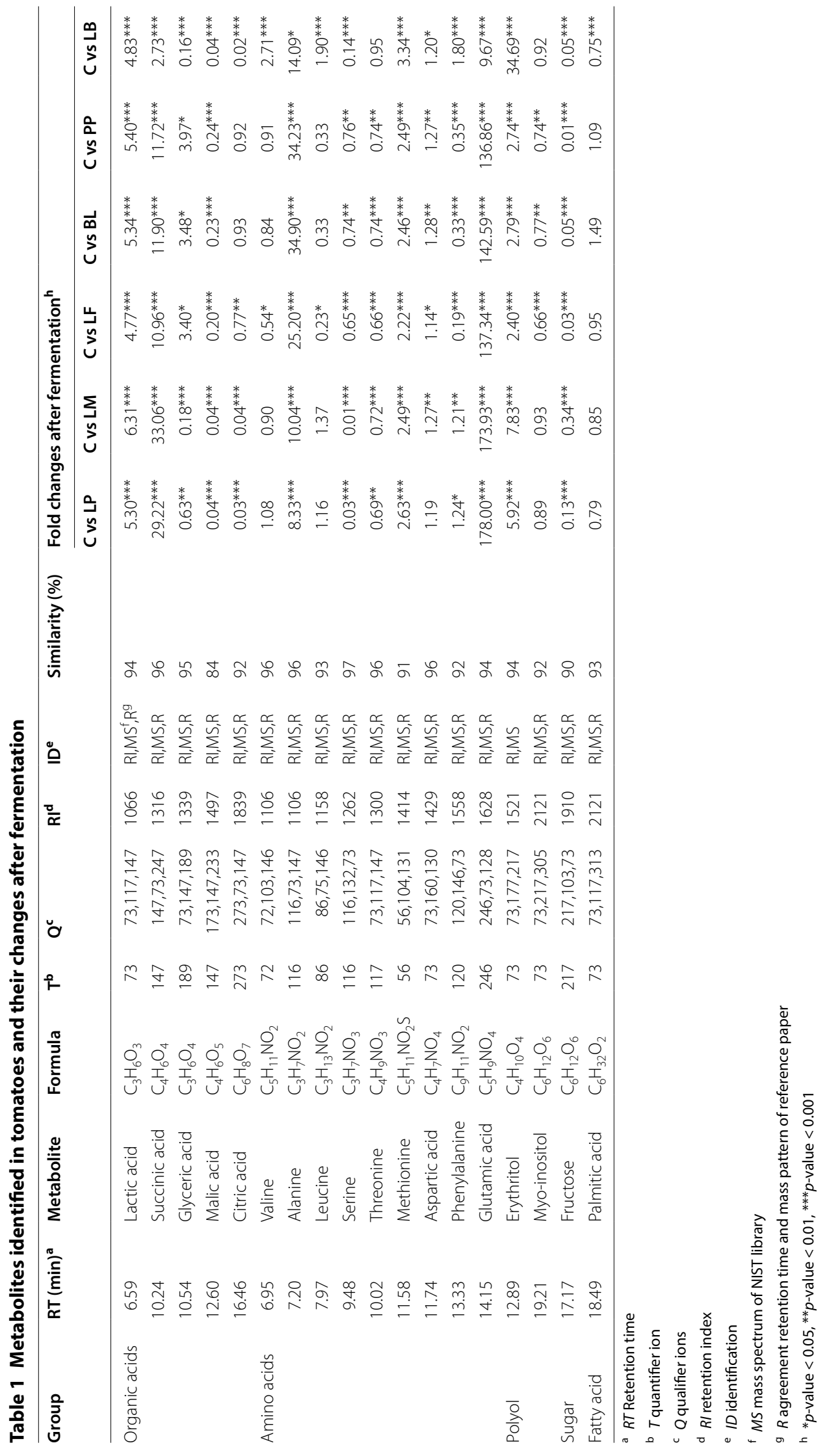


fermented with $L P$ and $L M$, which were not fully separated in score plot. The last group was tomato samples fermented with $L B$, implying that metabolites of tomato samples fermented with $L B$ were very different from the other samples. Interestingly, different or similar fermentation behaviors were observed for each LAB strain, even if the same tomato material was used.

\section{Metabolite differences of fermented tomatoes by different LABs}

To identify the effects of different LAB strains on metabolites identified in fermented tomatoes, PCA biplot was generated (Fig. 3). Similar to the results shown in Fig. 2b, PCA biplot showed clear separation into three groups. Two groups ( $L F, B L$, and $P P$ vs. $L P$ and $L M$ ) were clearly separated by PC1. Citric acid and malic acid were clustered in $L F, B L$, and $P P$. In addition, lactic acid, succinic acid, and fructose were related to $L P$ and $L M$ groups. Meanwhile, $L B$ was associated with erythritol on PC2.

Figure 4 shows the relative differences of the metabolites identified in tomatoes fermented by different $L A B$ strains. Tomatoes fermented by $L F, B L$, and $P P$ were found to have the highest levels of glyceric acid, malic acid, citric acid, alanine, and serine $(p<0.05)$, whereas levels of succinic acid and glutamic acid were significantly higher in tomatoes fermented by $L P$ and $L M$

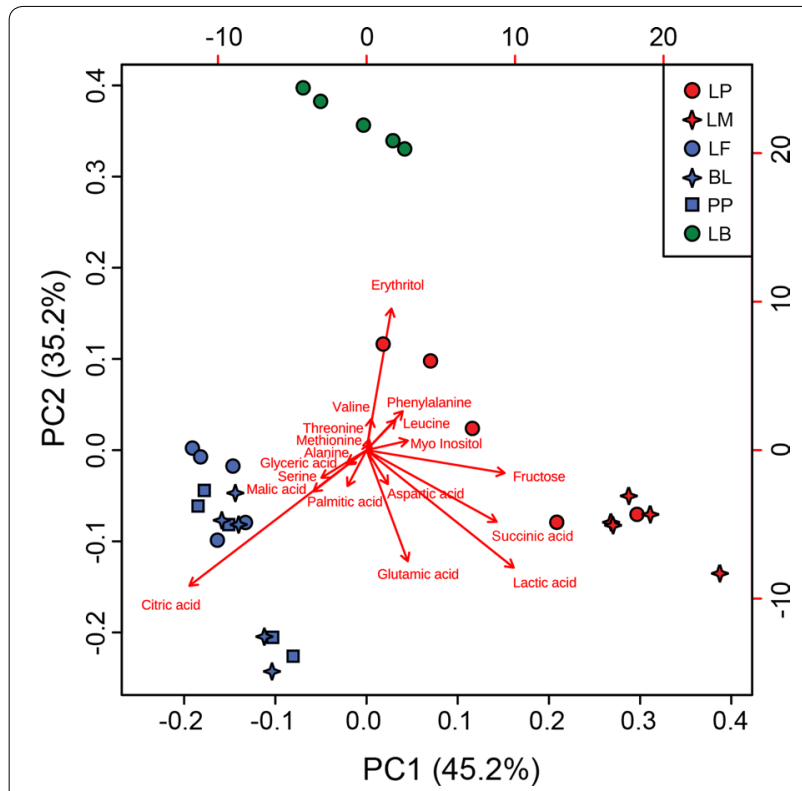

Fig. 3 PCA biplot derived from metabolites identified from tomato samples fermented with different $L A B$ strains. Arrows represent metabolites responsible for the divergence between tomatoes fermented with different LAB strains. LP Lactobacillus plantarum; LM Leuconostoc mesenteroid; LF Lactobacillus fermentum; BL Bifidobacterium longum; PP Pediococcus pentosaceus; LB Lactobacillus brevis $(p<0.05)$. The highest values of valine, leucine, threonine, methionine, phenylalanine, and erythritol were found in tomatoes fermented with $L B(p<0.05)$.

\section{Metabolic pathway analysis of LAB strains}

To determine the most relevant metabolic pathways affected by different LAB strains, metabolic pathway analyses were performed (Fig. 5). Metabolites identified in fermented tomato samples from the two most distinct groups ( $L F, B \mathrm{~L}$, and $P P$ vs. $L B$ ) were used for metabolic pathway analyses. Comparing $L F, B \mathrm{~L}$, and $P P$ versus $L B$ groups, impact values of aminoacyl-tRNA biosynthesis, cysteine and methionine metabolism, glycine, serine and threonine metabolism, citric acid metabolism, glutamine and glutamate metabolism, and glutathione metabolism were $0.18,0.12,0.25,0.12,0.17$, and 0.11 , respectively. On the basis of $p$ and impact values, aminoacyl-tRNA biosynthesis and metabolism of cysteine and methionine were identified as pathways affected by different $L A B$ groups ( $L F, B L$, and $P P$ vs. $L B$ groups).

\section{Discussion}

Lactic acid fermentation is a simple and widely used technique to enhance the nutritional value and shelflife of fruits and vegetables [20]. Several studies have reported changes in the quality of tomatoes fermented by LAB $[4,21]$. Since different metabolites can be produced depending on the $L A B$ strain used for fermentation [22], it is important to select appropriate LAB strains to fit the desired characteristics of the final product.

Although it is difficult to identify similarities or differences in metabolism between LAB strains, some studies have been conducted to identify differences between LAB strains using multivariate statistical analysis such as PCA. The PCA has been used for grouping the LAB strains, based on the morphological, physiological, and biochemical characteristics [23]. Roger et al. [24] reported that Kutukutu (fermented corn paste) must be fermented by L. brevis G25 and L. fermentum N33, based on the results of nutritional variables in the PCA. D'Angelo et al. [25] reported that the results of PCA score plot revealed three clusters of LAB strains regarding the behavior against the stress factors studied.

Recently, Metabolomics studies have been successfully used to display metabolic profiles of fermented foods predominated by LAB to predict fermentative characteristics of different microorganisms [26]. Some metabolomics studies have reported a clear separation between samples fermented with different LAB strains in PCA score plots obtained from fermented foods. According to Gallegos et al. [22], a PCA from GC-IMS spectral data of LAB allowed the differentiation of Lactobacillus and Lactococcus strains. In addition, this study presented the 
a
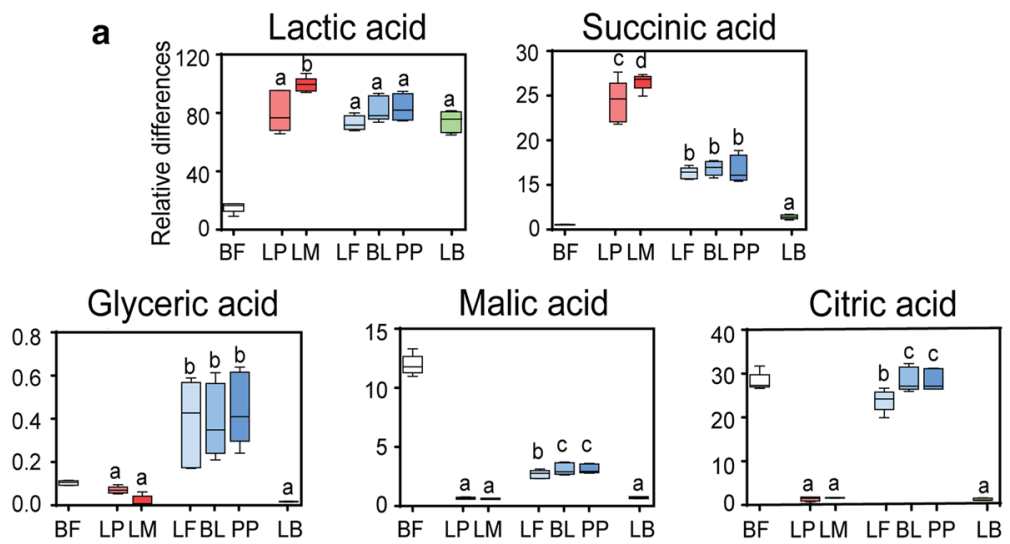

b

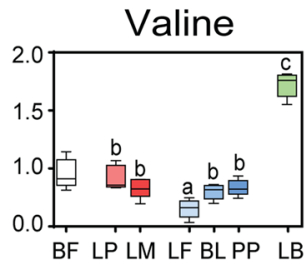

Serine

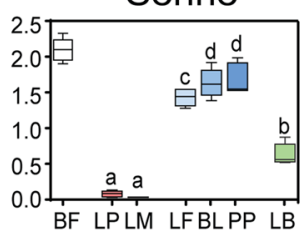

Aspartic acid

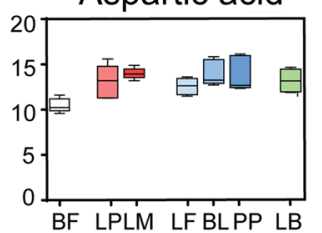

Alanine

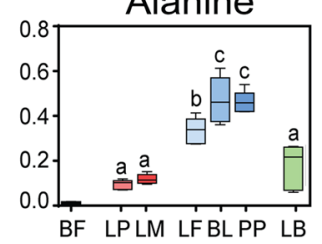

Threonine

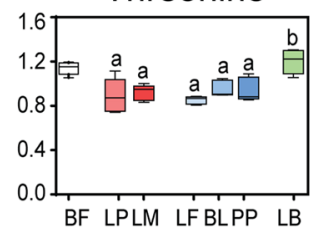

Phenylalanine

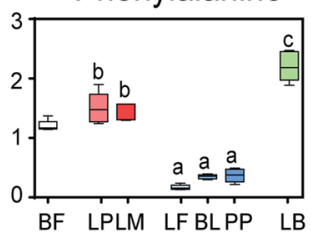

Leucine

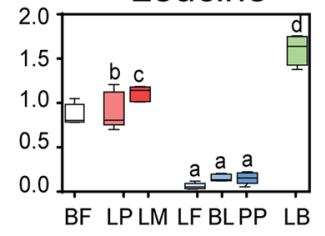

Methionine
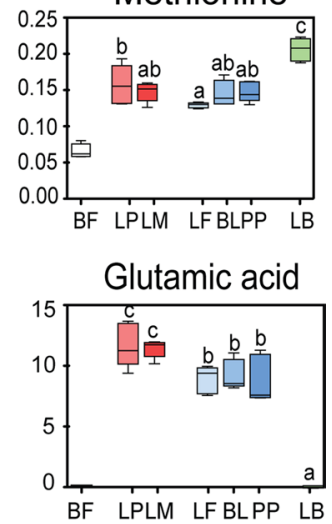

c

Erythritol

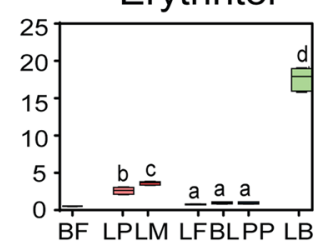

d

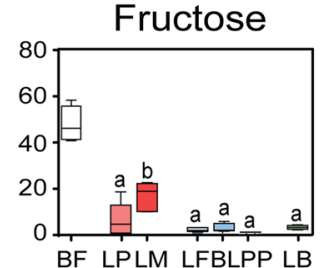

Myo-inositol

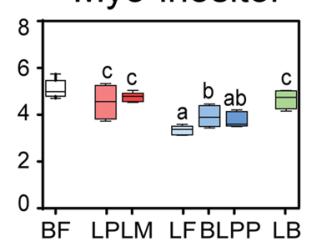

e

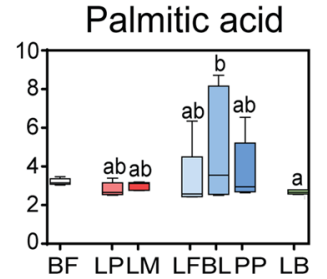

Fig. 4 Box plots of identified metabolites, including organic acids (a), amino acids (b), polyol (c), sugar (d), and fatty acid (e) in fermented tomatoes. Means followed by different letters are significantly different between samples $(p<0.05)$ 


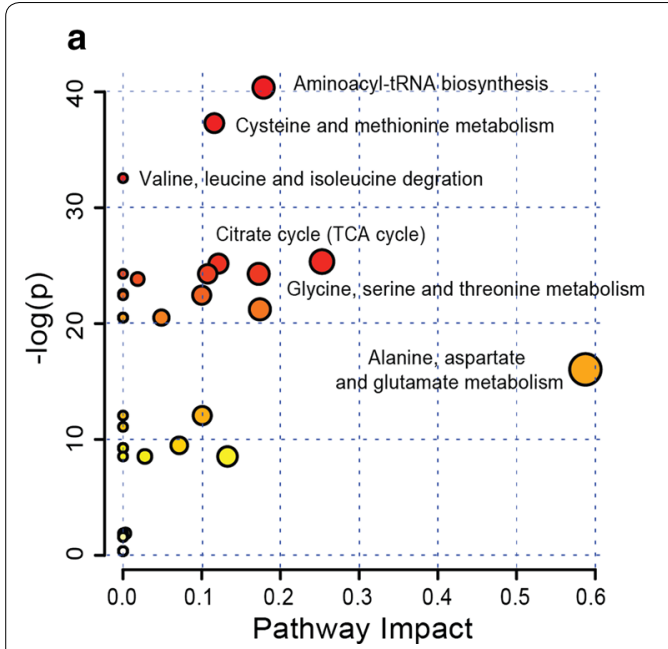

b

\begin{tabular}{|c|c|c|c|c|c|c|c|}
\hline $\begin{array}{l}\text { The } \\
\text { of cor }\end{array}$ & $\begin{array}{l}\text { number } \\
\text { mpounds }\end{array}$ & Hits & Raw $P$ & $-\log (P)$ & Holm adjust & FDR & Impact \\
\hline Aminoacyl-tRNA biosynthesis & 66 & 8 & $2.92 \mathrm{E}-18$ & $4.04 \mathrm{E}+01$ & $1.05 \mathrm{E}-16$ & $1.05 \mathrm{E}-16$ & 0.18 \\
\hline Cysteine and methionine metabolism & 37 & 3 & $6.30 \mathrm{E}-17$ & $3.73 E+01$ & $2.20 \mathrm{E}-15$ & 1.13E-15 & 0.12 \\
\hline $\begin{array}{l}\text { Valine, leucine and isoleucine } \\
\text { degration }\end{array}$ & 37 & 2 & $7.22 \mathrm{E}-15$ & $3.26 \mathrm{E}+01$ & $2.45 \mathrm{E}-13$ & $8.66 \mathrm{E}-14$ & 0.00 \\
\hline $\begin{array}{l}\text { Glycine, serine and } \\
\text { threonine metabolism }\end{array}$ & 28 & 4 & $9.74 \mathrm{E}-12$ & $2.54 \mathrm{E}+01$ & $3.22 \mathrm{E}-10$ & $8.37 \mathrm{E}-11$ & 0.25 \\
\hline Citric acid (TCA cycle) & 20 & 2 & 1.16E-11 & $2.52 E+01$ & $3.72 \mathrm{E}-10$ & $8.37 \mathrm{E}-11$ & 0.12 \\
\hline $\begin{array}{l}\text { D-Gluamine and D-Glutamate } \\
\text { metabolism }\end{array}$ & 7 & 1 & $2.81 \mathrm{E}-11$ & $2.43 \mathrm{E}+01$ & $8.72 \mathrm{E}-10$ & 1.27E-10 & 0.17 \\
\hline Glutathione metabolism & 17 & 1 & $2.81 \mathrm{E}-11$ & $2.43 E+01$ & $8.72 \mathrm{E}-10$ & $1.27 \mathrm{E}-10$ & 0.11 \\
\hline
\end{tabular}

Fig. 5 Metabolic pathway (a) generated using MetaboAnalyst to identify the most relevant pathways from metabolites identified in fermented tomato samples between $\angle F, B L$, and $P P$ versus $\angle B$. The color and size of the circles indicate significant changes in metabolites and the impact score in the metabolic pathways, respectively. Panel (b) shows statistics for major changed pathways, based on $p$ value and false discovery rate. $L F$ Lactobacillus fermentum, BL Bifidobacterium longum, PP Pediococcus pentosaceus, LB Lactobacillus brevis

possibility of discrimination Lactobacillus casei from Lactoabillus paracasei, according to their relevant volatile fingerprints. Correia et al. [27] have also reported that sorghum fermented with $L B$ was very different from samples fermented with $L P, L F$, and $P P$, due to the superior contents of specific macromolecules of the proteins and lipids. However, little is known about the similarities or differences in metabolic characteristics between LAB strains.

Results of PCA analyses in the present study indicated that metabolite profiles of tomatoes were dependent on the LAB strain used. Since metabolites of tomatoes are related to taste and flavor $[28,29]$, differences in metabolites, depending on the LAB strain, indicate differences in quality. For example, the umami of tomato has been related to the content of amino acids such as citric acid, glycine, serine and glutamic acid [30]. Among them, glutamic acid played an important role as umami in tomato taste [31]. It has been also well known that the sugars such as glucose and fructose of tomatoes affect sweetness. The sour taste in tomatoes was attributed mainly to citric and malic acids [32]. The high levels of lactic acid in tomato fermented with $L M$ can be associated with the sour taste. Further metabolomics studies involving more samples are required to clarify the relationship between taste and LAB strains.

\section{Author's contributions}

E-JK conceived of the presented idea and planned the experiments. O-CK provided materials for experiment. S-HS conducted all statistical analyses. E-JK and S-EP wrote the article. H-SS supervised the project. All authors read and approved the final manuscript.

\section{Author details}

${ }^{1}$ School of Korean Medicine, Dongshin University, Naju 58245, Republic of Korea. ${ }^{2}$ Gwangsu Farming Association, Damyang 57368, Republic of Korea.

\section{Acknowledgements}

This research was financially supported by the Ministry of SMEs and Startups (MSS), Korea, under the "Regional Specialized Industry Development Program (R\&D, R0006327)" supervised by the Korea Institute for Advancement of Technology (KIAT).

\section{Competing interests}

The authors declare that they have no competing interests.

\section{Publisher's Note}

Springer Nature remains neutral with regard to jurisdictional claims in published maps and institutional affiliations.

Received: 8 November 2018 Accepted: 4 February 2019

Published online: 20 February 2019

\section{References}

1. Abushita AA, Daood HG, Biacs PA (2000) Change in carotenoids and antioxidant vitamins in tomato as a function of varietal and technological factors. J Agric Food Chem 48:2075-2081

2. Jang SY, Woo SM, Jo YJ, Kim OM, Kim IH, Jeong YJ (2010) Quality characteristics of tomato wine on fermentation conditions. J Korean Soc Food Sci Nutr 39:443-448

3. Story EN, Kopec RE, Schwartz SJ, Harris GK (2010) An update on the health effects of tomato lycopene. Annu Rev Food Sci Technol 1:189-210

4. Cagno RD, Coda R, Angelis MD, Gobbetti M (2013) Exploitation of vegetables and fruits through lactic acid fermentation. Food Microbiol 33:1-10

5. Mechmeche M, Kachouri F, Ksontini H, Hamdi M (2017) Production of bioactive peptides from tomato seed isolate by Lactobacillus plantarum fermentation and enhancement of antioxidant activity. Food Technol Biotechnol 31:94-113

6. Moayedi A, Hashemi M, Safari M (2016) Valorization of tomato waste proteins through production of antioxidant and antibacterial hydrolysates by proteolytic Bacillus subtilis: optimization of fermentation conditions. J Food Sci Technol 53:391-400 
7. Koek MM, Muilwijk B, Werf MJVD, Hankemeier T (2006) Microbial metabolomics with gas chromatography/mass spectrometry. Anal Chem 78:1272-1281

8. Trushina E, Mielke MM (2014) Recent advances in the application of metabolomics to Alzheimer's disease. Biochim Biophys Acta Mol Basis Dis 1842:1232-1239

9. Kazimierczak R, Hallmann E, Lipowski J, Drela N, Kowalik A, Püssa T, Matt D, Luik A, Gozdowski D, Rembialkowska E (2004) Beetroot (Beta vulgaris L.) and naturally fermented beetroot juices from organic and conventional production: metabolomics, antioxidant levels and anticancer activity. J Sci Food Agric 94:2618-2629

10. Janningsmeier SD, McFeeters RF (2015) Metabolic footprinting of Lactobacillus buchneri strain LA1147 during anaerobic spoilage of fermented cucumbers. Int J Food Microbiol 215:40-48

11. Filannino P, Cardinali G, Rizzello CG, Buchin S, Angelis MD, Gobbetti M, Cagno RD (2014) Metabolic responses of Lactobacillus plantarum strains during fermentation and storage of vegetable and fruit juices. Appl Environ Microbiol 80:2206-2215

12. Cagno RD, Surico RF, Paradiso A, Angelis MD, Salmon JC, Buchin S, Gara LD, Gobbetti M (2009) Effect of autochthonous lactic acid bacteria starters on health-promoting and sensory properties of tomato juices. Int J Food Microbiol 128:473-483

13. Takeda S, Matsufuji H, Nakade K, Takenoyama SI, Ahhmed A, Sakata R, Kawahara S, Muguruma M (2017) Investigation of lactic acid bacterial strains for meat fermentation and the product's antioxidant and angiotensin-I-converting-enzyme inhibitory activities. Anim Sci J 88:507-516

14. Folin O, Denis W (1912) On phosphotungstic-phosphomolybdic compounds as color reagents. J Biol Chem 12:239-243

15. Zhishen J, Mengcheng T, Jianming W (1999) The determination of flavonoid contents in mulberry and their scavenging effects on superoxide radicals. Food Chem 64:555-559

16. Blois MS (1958) Antioxidant determinations by the use of a stable free radical. Nature 181:1199-1200

17. Mastrangelo A, Ferrarini A, Rey-Stolle F, García A, Barbas C (2015) From sample treatment to biomarker discovery: a tutorial for untargeted metabolomics based on GC-(EI)-Q-MS. Anal Chim Acta 900:21-35

18. Park SE, Seo SH, Kim EJ, Byun SH, Na CS, Son HS (2019) Changes of microbial community and metabolite in kimchi inoculated with different microbial community starters. Food Chem 274:558-565

19. Kwaw E, Ma Y, Tchabo W, Apaliya MT, Wu M, Sackey AS, Xiao L, Tahir HE (2018) Effect of lactobacillus strains on phenolic profile, color attributes and antioxidant activities of lactic-acid-fermented mulberry juice. Food Chem 250:148-154

20. Merabti R, Bekhouche F, Chuat V, Madex MN, Maiilard MB, Bailly S, Thierry A, Valence $F$ (2015) A large diversity of lactic acid bacteria species is involved in the fermentation of wheat used for the manufacture of lemzeiet. Eur Food Res Technol 214:137-149

21. Piasecka-Jóźwiak K, Rozmierska J, Chablowska B, Stecka KM, Skapska S, Kliszcz M, Szkudzińska-Rzeszowiak S (2013) Starter cultures for lactic acid fermentation of sweet pepper, pattypan squash and tomatoes. Pol J Food Nutr Sci 63:95-102

22. Gallegos J, Arce C, Jordano R, Arce L, Medina LM (2017) Target identification of volatile metabolites to allow the differentiation of lactic acid bacteria by gas chromatography-ion mobility spectrometry. Food Chem 220:362-370

23. Mugula JK, Nnko SA, Narvhus JA, Sorhaug T (2003) Microbiological and fermentation characteristics of togwa, a Tanzanian fermented food. Int Food Microbiol 80:187-199

24. Roger T, Léopold TN, Funtong MMC (2015) Nutritional properties and antinutritional factors of corn paste (Kutukutu) fermented by different strains of lactic acid bacteria. Int J Food Sci 2015:1-13

25. D’Angelo L, Cicotello J, Zago M, Guglielmotti D, Quiberoni A, Suarez V (2017) Leuconostoc strains isolated from dairy products: response against food stress conditions. Food Microbiol 66:28-39

26. Mozzi F, Ortiz ME, Bleckwedel J, Vuyst LD, Pescuma M (2013) Metabolomics as a tool for the comprehensive understanding of fermented and functional foods with lactic acid bacteria. Food Res Int 54:1152-1161

27. Correia I, Nunes A, Guedes S, Barros AS, Delgadillo I (2010) Screening of lactic acid bacteria potentially useful for sorghum fermentation. J Cereal Sci 52:9-15

28. Bellens K, Mészáros P, Vermeir S, Kirsanow D, Legin A, Buysens S, Cap N, Nicolaï BM, Lammertyn L (2008) Analysis of tomato taste using two types of electronic tongues. Sens Actuator B Chem 131:10-17

29. Yilmaz E (2001) The chemistry of fresh tomato flavor. Turk J Agric For 25:149-155

30. Zushi K, Matsuzoe N (2011) Utilization of correlation network analysis to identify differences in sensory attributes and organoleptic compositions of tomato cultivars grown under salt stress. Sci Hortic 129:18-26

31. Fuke S, Shimizu T (1993) Sensory and preference aspects of umami. Trends Food Sci Technol 4:246-251

32. Petro-Turza M (1986) Flavor of tomato and tomato products. Food Rev Int 2:309-351

\section{Submit your manuscript to a SpringerOpen ${ }^{\odot}$ journal and benefit from:}

- Convenient online submission

- Rigorous peer review

- Open access: articles freely available online

- High visibility within the field

Retaining the copyright to your article

Submit your next manuscript at springeropen.com 